\title{
Cuidado ao paciente em ecmo (extracorporeal membrane oxygenation): um desafio para a enfermagem neonatal
}

\begin{abstract}
RESUMO | Objetivo: avaliar as evidências científicas disponíveis sobre as intervenções de enfermagem no atendimento ao recém-nascido submetido à terapia de oxigenação por membrana extracorpórea. Método: Pesquisa bibliográfica utilizando como fonte de pesquisa plataformas online como Ovid MEDLINE, EMBASE, Cochrane Central Register of Controlled Trials e Pubmed, SCOPUS, Scielo nos últimos 15 anos, realizada no período de junho de 2019 à outubro de 2020. Resultado: a utilização da membrana de oxigenação extracorpórea é uma terapia de alta complexidade, cabendo ao enfermeiro, privativamente, a responsabilidade pela assistência direta ao paciente submetido a esta intervenção. É primordial que o enfermeiro possua amplo conhecimento técnico-científico sobre o procedimento a fim de garantir a qualidade da assistência de enfermagem e a segurança do paciente em uso da tecnologia em estudo. Conclusão: O conhecimento científico e a capacitação profissional são vistos como fatores determinantes para o êxito na implantação e manutenção da oxigenação por membrana extracorpórea.
\end{abstract}

Descritores: Membrana de oxigenação extracorpórea; Neonatologia; Cuidados de enfermagem.

\begin{abstract}
Objective: to evaluate the available scientific evidence on nursing interventions in the care of newborns submitted to extracorporeal membrane oxygenation therapy. Method: Literature search using online platforms such as Ovid MEDLINE, EMBASE, Cochrane Central Register of Controlled Trials and Pubmed, SCOPUS, Scielo in the last 15 years, carried out between June 2019 and October 2020. Result: the use of extracorporeal membrane oxygenation is a highly complex therapy, and the nurse is privately responsible for the direct assistance to the patient undergoing this intervention. It is essential that nurses have extensive technical- scientific knowledge about the procedure in order to ensure the quality of nursing care and patient safety using the technology under study. Conclusion: Scientific knowledge and professional training are seen as determining factors for the successful implementation and maintenance of extracorporeal membrane oxygenation.
\end{abstract}

Descriptors: Extracorporeal oxygenation membrane; Neonatology; Nursing care.

RESUMEN | Objetivo: evaluar la evidencia científica disponible sobre las intervenciones de enfermería en el cuidado del recién nacido sometido a terapia de oxigenación por membrana extracorpórea. Método: Búsqueda de literatura utilizando plataformas en línea como Ovid MEDLINE, EMBASE, Registro Cochrane Central de Ensayos Controlados y Pubmed, SCOPUS, Scielo en los últimos 15 años, realizada desde junio de 2019 hasta octubre de 2020. Resultado: el uso de oxigenación por membrana extracorpórea es una terapia de alta complejidad, siendo la enfermera la responsable privada de la asistencia directa al paciente sometido a esta intervención. Es fundamental que el enfermero cuente con un amplio conocimiento técnico-científico sobre el procedimiento para garantizar la calidad de la atención de enfermería y la seguridad del paciente utilizando la tecnología en estudio. Conclusión: El conocimiento científico y la formación profesional se ven como factores determinantes para la implementación y el mantenimiento exitosos de la oxigenación por membrana extracorpórea.

Descriptores: Membrana de oxigenación extracorpórea; Neonatología; Cuidado de enfermera.

\section{Tamara Soares}

Enfermeira, Mestre em Enfermagem, Enfermeira da UTI Neonatal do Hospital de Clínicas de Porto Alegre.

ORCID: 0000-0001- 5579-7510

\section{Ana Cristina Nunes Salvado}

Enfermeira, Especialista em Enfermagem Hospitalar à Criança e ao Adolescente, Enfermeira da UTI Neonatal do Hospital de Clínicas de Porto Alegre.

ORCID: 0000-0003-0504-2501

\section{Cristiane Raupp Nunes}

Enfermeira, Doutora em Saúde da Criança e do Adolescente, Enfermeira da UTI Neonatal do Hospital de Clínicas de Porto Alegre.

ORCID: 0000-0001-8818-3072

\section{Maitê Larini Rimolo}

Enfermeira, Especialista em Enfermagem em Neonatologia, Enfermeira da UTI Neonatal do Hospital de Clínicas de Porto Alegre.

ORCID: 0000-0001-9447-8539

\section{Raquel Cristina Concatto}

Enfermeira, Mestre em Saúde da Criança e do Adolescente, Enfermeira da UTI Neonatal do Hospital de Clínicas de Porto Alegre. ORCID: 0000-0001-5681-3893
Recebido em: 27/08/2021

Aprovado em: 30/11/2021 
INTRODUÇÃO

A Oxigenação por Membrana Extracorpórea (termo em inglês, Extracorporeal Membrane Oxygenation - ECMO) é uma terapia que usa circulação extracorpórea parcial modificada com membrana de oxigenação para prestar apoio pulmonar e/ou cardíaco. É usada em pacientes com insuficiência cardiopulmonar reversível de causas pulmonares, cardíacas ou de outro tipo1,2.

Em 1975, no Orange County Medical Center, Bartlett usou a ECMO com sucesso em um recém-nascido latino abandonado que sofria de síndrome do desconforto respiratório, tendo seu uso em neonatos aumentado no fim dos anos 1980. Devido a esse aumento, uma aliança de voluntários, a Organização de Apoio Extracorpóreo à Vida, foi formada entre centros de ECMO, em 19892.

Nas unidades de terapia intensiva neonatal, a ECMO está sendo utilizada no auxílio ao tratamento de alguns problemas cardiorrespiratórios, tais como síndrome de aspiração de mecônio, hipertensão pulmonar persistente do recém-nascido, hérnia diafragmática congênita, sepses/pneumonia, síndrome do desconforto respiratório e insuficiência cardiopulmonar3,4.

Nos últimos anos, com o surgimento de novas terapias respiratórias, como o uso de óxido nítrico inalatório, de surfactante pulmonar e de ventilação oscilatória de alta frequência, a ECMO tem sido utilizada apenas após criteriosa avaliação, visto que é um procedimento altamente invasivo associado a muitos riscos e com alto custo. Quando utilizada é uma terapia de resgate para muitas doenças neonatais, casos mais complexos e de maior gravidade da doença primária, onde os tratamentos convencionais não surtiram efeito, garantindo assim sua superioridade em relação a muitos tratamentos convencionais como relatam alguns estudos $3,4,5$
Conforme mostrado em um estudo multicêntrico controlado e randomizado realizado no Reino Unido com 185 recém-nascidos com insuficiência res-

A Oxigenação por Membrana Extracorpórea (termo em inglês, Extracorporeal Membrane Oxygenation $\mathrm{ECMO}$ ) é uma terapia que usa circulação extracorpórea parcial modificada com membrana de oxigenação para prestar apoio
pulmonar e/ou
cardíaco

piratória grave em 55 hospitais, a mortalidade e a deficiência grave avaliadas no $1^{\circ}, 4^{\circ}$ e $7^{\circ}$ anos de vida reduziram significativamente com o uso da ECMO
(59\% para o grupo de terapia convencional em comparação com 37\% para o grupo de ECMO) 6 .

O uso da ECMO em neonatos próximos ao termo com insuficiência respiratória grave, porém possivelmente reversível, melhora significativamente a sobrevida sem aumentar deficiências graves e tem a melhor relação custo/ benefício em comparação com outras terapias de tratamento intensivo7,8.

Na última década, a ECMO foi utilizada, anualmente, como terapia de suporte em cerca de 800 neonatos que não respondiam ao tratamento intensivo com ventilação oscilatória de alta frequência (VOAF) e/ou inalação de óxido nítrico (NO). Atualmente, a taxa de uso da ECMO nos EUA é de aproximadamente um neonato a cada 5.000 nascidos vivos 1 .

Partindo do pressuposto que a enfermagem exerce um importante papel no cuidado ao paciente sob este tipo de assistência, propôs-se a presente investigação com o objetivo de avaliar, através de uma revisão da literatura, as evidências disponíveis na literatura sobre as intervenções de enfermagem eficazes para o atendimento ao recém-nascido submetido à intervenção ECMO.

\section{METODOLOGIA}

O presente estudo caracteriza-se como uma pesquisa bibliográfica, buscando conhecer sob o olhar de diversos autores, o desafio para equipe de enfermagem na assistência para os pacientes em ECMO na Unidade de Terapia Intensiva Neonatal.

A pesquisa bibliográfica consiste no exame de produções desenvolvidas ao longo da evolução da humanidade, para levantamento e análise do que já se produziu sobre determinado assunto que são assumidos como tema de pesquisa científica. Dessa forma, a pesquisa bibliográfica propicia o exame de um tema sob novo enfoque ou abordagem, chegando a conclusões próprias9. 
Para o desenvolvimento do estudo em questão, no período de junho de 2019 a outubro de 2020, buscou-se, como fonte de pesquisa, plataformas online como Ovid MEDLINE, EMBASE, Cochrane Central Register of Controlled Trials e Pubmed, SCOPUS, Scielo. Os descritores em inglês utilizados foram: Extracorporeal membrane oxygenation AND neonatal AND nursing care. A consulta foi limitada aos últimos 15 anos, com pesquisas em humanos e texto completo (full text). Foram incluídos na amostra os artigos que se enquadraram nos seguintes critérios: artigos sem restrição de idiomas, com seus resumos publicados nas bases de dados selecionadas, no período compreendido entre junho de 2005 a maio de 2020. Além disso, levou-se em consideração que a metodologia adotada permitisse obter evidências científicas acerca desta terapêutica, sendo assim, foram incluídos ensaios clínicos randomizados controlados individualmente ou estudos com delineamento de pesquisa quase experimental e artigos que retratassem procedimentos, intervenções que contribuíssem para responder à questão norteadora. Foram excluídos estudos do tipo revisão de literatura ou sistemática, cartas e editoriais, teses, dissertações, trabalhos de conclusão de curso, artigos não referentes ao período neonatal.

Para a organização do material, foi utilizado o sistema de fichamento dos materiais obtidos a fim de dinamizar o desenvolvimento da pesquisa. Com o material selecionado, buscou-se contemplar os objetivos propostos e com isso realizar o levantamento dos cuidados de enfermagem ao paciente em ECMO na UTI Neonatal. RESULTADOS

A primeira busca resultou em 351 artigos dos quais, após aplicar os critérios de exclusão e a realização da leitura dos resumos, identificamos 28 publicações pertinentes com a questão norteadora da pesquisa. Da totalidade dos artigos, sob a ótica do idioma, 25\%

dos selecionados estão publicados em português, 67,9\% em inglês e 7,1\% em espanhol. Em relação ao ano de publicação, 60,7\% dos trabalhos foram pu-

Durante a terapia, o sangue é drenado por uma cânula na veia do paciente para uma bomba externa (rotativa ou centrífuga), que circula o sangue por meio de uma membrana de troca loxigenador de silicone ou polimetilpentano) para oxigenação e remoção do $\mathrm{CO} 2$ e de um aquecedor para devolver o sangue à circulação
do paciente

blicados nos últimos 5 anos.

\section{DISCUSSÃO}

Após leitura total dos artigos sele- cionados, as informações foram seccionadas conforme os títulos: fisiologia da ECMO, indicações e contraindicações para ECMO, equipe multidisciplinar, a assistência de enfermagem e complicações.

Fisiologia da ecmo

ECMO é uma terapia aplicada em casos de falência respiratória na qual o sangue é oxigenado fora do corpo do paciente. Esse processo aumenta a troca de gases, além de permitir o ajuste de parâmetros ventilatórios potencialmente nocivos ao parênquima pulmonar10. A oxigenação por membrana extracorpórea proporciona "tempo para descanso" e recuperação pulmonar e cardíaca6.

Durante a terapia, o sangue é drenado por uma cânula na veia do paciente para uma bomba externa (rotativa ou centrífuga), que circula o sangue por meio de uma membrana de troca (oxigenador de silicone ou polimetilpentano) para oxigenação e remoção do $\mathrm{CO} 2$ e de um aquecedor para devolver o sangue à circulação do paciente. Essa terapia exige a anticoagulação do circuito e do paciente com heparina administrada ao sistema da ECMO, a fim de evitar a ativação da cascata de coagulação. Além disso, diversos monitores de pressão, fluxo, bolhas e temperatura são usados. O monitoramento contínuo da coagulação é fundamental com o uso de tempo de coagulação ativada (TCA), nível de antifator $\mathrm{Xa}$, contagem de plaquetas, medição dos níveis de fibrinogênio e, em alguns pacientes, nível de antitrombina III e tromboelastografia1.

A terapia possui duas modalidades, a veno-arterial (VA) que permite suporte hemodinâmico e ventilatório onde o sangue é drenado por uma cânula venosa e retorna pela cânula arterial. O modo veno-venoso (VV) permite a troca gasosa, utilizando duas cânulas venosas11.

Após o sucesso do procedimento 
de 1975, vários estudos relataram uma sobrevida significativa de recém-nascidos submetidos à ECMO por insuficiência respiratória grave reversível por consequência de várias patologias, tornando a ECMO nessa faixa etária uma opção de tratamento padrão12,13,14. No Brasil, seu uso está em ascensão na assistência circulatória mecânica, principalmente em hospitais com atendimento de alta complexidade15.

Indicações e contra indicações para ecmo

Atualmente não há critérios fixos para o atendimento com ECMO em neonatologia. A maioria dos centros indicam recém-nascidos com idade gestacional superior a 34 semanas, devido ao alto risco de hemorragia intracraniana consequente do uso de anticoagulantes. Por conta do tamanho das cânulas disponíveis, a terapia é indicada a recém-nascidos com peso acima de dois quilos. Recém-nascidos asfixiados ou que necessitem hipotermia terapêutica podem atender aos critérios da ECMO, atentando para evitar complicações da coagulação causadas pelo processo de resfriamento e heparinização. Além dos critérios para seleção, são aplicados critérios clínicos de qualificação específicos6,13,16. As indicações pulmonares em neonatos são: doença da membrana hialina, síndrome da aspiração do mecônio, hipertensão pulmonar primária do recém-nascido, hérnia diafragmática congênita, sepse neonatal, pneumonia e outras patologias que cursam com hipertensão pulmonar17.

As contraindicações são: recém-nascidos com doenças pré-existentes como hemorragia intracraniana; alguma forma de coagulopatia; anormalidades congênitas ou cromossômicas letais; e lesão cerebral irreversível ou cardiopatia congênita6.

O descumprimento dos critérios de seleção e indicações citados acima para oxigenação por membrana ex-

tracorpórea são uma contraindicação para o uso da ECMO, além de patologias irreversíveis e pré-existentes que prejudicam a qualidade de vida18.

Atualmente não há critérios fixos para o atendimento com ECMO em neonatologia. A maioria dos centros indicam recémnascidos com idade gestacional superior

a 34 semanas, devido ao alto risco de hemorragia intracraniana consequente do uso de anticoagulantes

\section{Equipe multidisciplinar}

De acordo com a ELSO, o especialista em ECMO é "o profissional treinado para lidar com o equipamen- to e com as necessidades clínicas do paciente sob a direção e supervisão de um médico treinado em ECMO"1. Dessa forma, cada vez mais profissionais buscam o aperfeiçoamento nessa técnica e o enfermeiro é o profissional que lida com a assistência direta ao paciente submetido a esta intervenção.

Os cuidados multidisciplinares de pacientes em ECMO em Unidade de Terapia Intensiva são importantes para providenciar uma assistência bem delimitada. O procedimento de canulação é crítico porque os pacientes admitidos em ECMO estão em condições graves, às vezes exigindo manobras de reanimação e a há a necessidade de uma ligação rápida ao circuito para iniciar a terapia. Há necessidade de velocidade, coordenação e ação simultânea das equipes envolvidas. As habilidades técnicas dos profissionais envolvidos, suprimentos e equipamentos adequados disponíveis e protocolos e listas de verificação disponíveis contribuem ao sucesso da canulação e do início da terapia19. ECMO exige um alto nível de habilidades técnicas e não-técnicas, associadas a um time multidisciplinar altamente capacitado contemplando médicos, enfermeiros e perfusionistas20.

A assistência de enfermagem

O processo de enfermagem, a monitoração, a administração de medicamentos e revisão do circuito constituem algumas das tarefas em que o enfermeiro dispende um tempo considerável e que tem papel essencial nesta terapia19. A assistência de enfermagem ao paciente com ECMO deve ser realizada mediante a elaboração do Processo de Enfermagem e com base em um protocolo institucional que padronize os cuidados prestados21. O uso de ECMO é uma realidade e deve sempre ser levado em conta um cuidado de enfermagem integral e individualizado onde todas as necessidades do paciente são consideradas19. O que consolida um 
grande desafio para o enfermeiro supri-las dentro de uma realidade onde ainda há escassez de recursos humanos capacitados.

As competências mínimas necessárias para o profissional de enfermagem que trabalha com ECMO são: identificar tipo de ECMO VV ou VA, conhecer os componentes do circuito de ECMO, definir e demonstrar como tirar o paciente do ECMO em uma emergência, identificar potenciais emergências e como lidar com elas, identificar os equipamentos necessários ao lado do leito, apresentar técnicas de resoluções de problemas, identificar e descrever a decanulação, verbalizar o entendimento de quando notificar o especialista em ECMO19.

No momento da definição da indicação da terapia a equipe médica realiza contato com o cirurgião pediátrico responsável pela canulação e com a equipe de perfusionistas. A enfermeira providencia material e notifica o banco de sangue para providenciar os hemoderivados prescritos, pela equipe médica, para preenchimento do sistema. Como logística ideal, previamente já estará definido um leito na unidade onde a ECMO será realizada. Caso o paciente não esteja neste leito, a enfermeira providencia a transferência para este leito e certifica de que todo material, antes listado, esteja acessível para uso imediato. A enfermagem está presente durante todo o processo que envolve a terapia prestando cuidados complexos que demandam estrutura física, equipamentos, monitoramento e atenção contínua das condições clínicas do paciente22. A padronização dos locais dos equipamentos faz parte da organização da unidade do paciente e garante a segurança de todo processo. A observação é constante no circuito para identificação da formação de trombos, com o auxílio de um foco luminoso. Outro aspecto importante é a prevenção de lesões de pele, controle glicêmico, hídrico, térmico e do nível

de consciência15. Cabe, privativamente, ao enfermeiro a responsabilidade pela assistência direta ao paciente submetido a esta intervenção23. À medi-

No momento da definição da indicação da terapia a equipe médica realiza contato com o cirurgião pediátrico responsável pela canulação e com a equipe de perfusionistas. A enfermeira providencia material e notifica o banco de sangue para providenciar os hemoderivados prescritos, pela equipe médica, para preenchimento do sistema

da que o paciente melhora, o suporte da ECMO é gradualmente reduzido. Os pacientes são decanulados quando não podem tolerar o suporte mínimo da
ECMO com parâmetros baixos a moderados de ventilação mecânica. O tratamento com ECMO normalmente dura entre cinco e dez dias para pacientes neonatais com doenças respiratórias e mais tempo em casos de hérnia diafragmática congênita (10 a 12 dias em média)1.

Complicações

A complicação mais comum da ECMO é de longe a necessidade de drogas vasoativas durante o apoio extracorpóreo, seguido de hemorragia do sítio cirúrgico6.

As complicações mecânicas (decorrentes do circuito) mais comuns são coágulos no circuito, coagulopatia de consumo e embolias. A canulação pode causar danos na veia jugular, podendo ocasionar hemorragia massiva do mediastino e lesão na artéria carótida que pode gerar uma dissecção da aorta13,24,25. As falhas do circuito que podem ocorrer são com a fonte de oxigênio e misturadores de oxigênio, falha dos equipamentos de monitoramento, rachaduras nos conectores, ruptura de tubo e mau funcionamento da bomba, podendo ser apenas um retorno venoso inadequado22.

As complicações clínicas normalmente são consequências da anticoagulação, das interações do sangue com as superfícies artificiais do circuito e das mudanças no padrão de fluxo sanguíneo. Podem ocorrer complicações neurológicas, hemorrágicas, cardíacas, pulmonares e renais26,27,28.

A hemorragia intracraniana é a principal causa de óbito durante o ECMO e o aparecimento de convulsões é um sinal de mau prognóstico. Também existem complicações de falhas do circuito do oxigenador ou de outros equipamentos da ECMO1.

\section{CONCLUSÃO}

Embora muito progresso tenha sido feito nesse campo e apesar dos dife- 
rentes modelos estudados, ainda há a necessidade do desenvolvimento de novas produções cientificas sobre o tema, para mostrar que a capacidade cardiopulmonar está protegida e continua a amadurecer durante o suporte de ECMO.

Uma limitação para a construção desse artigo é que boa parte dos artigos pesquisados sobre o tema contém estudos e revisões bibliográficas que envolvem artigos antigos de mais de 20 anos, corroborando com o que afirma- mos anteriormente sobre as produções intelectuais sobre ECMO especialmente em pacientes neonatais.

Apesar da escassez de material é perceptível a importância do enfermeiro no uso dessa técnica que tende a crescer fortemente no Brasil. O conhecimento científico e a capacitação profissional são vistos como fatores determinantes para o êxito na implantação e manutenção da oxigenação por membrana extracorpórea.

Podemos concluir que o conhe- cimento sobre o assunto ainda é uma barreira a ser vencida pela equipe assistencial, mas além disso, as habilidades técnicas são de extrema importância para a equipe, sendo assim, torna-se seguro afirmar que estes são os principais obstáculos a serem enfrentados pela equipe de enfermagem quando se trata de uma proposta de cuidado tão inovadora e desafiadora quanto é a ECMO.

\section{Referências}

1. ELSO Data Registry. ECMO Registry of the Extracorporeal Life Support Organization (ELSO). Ann Arbor: University of Michigan; 2016. Available from: http://www.elso.org [cited 21/08/2020]. [ Links ]

2. FORTENBERRY, James D.; LORUSSO, Roberto. The history and development of extracorporeal support. Extracorporeal life support, v. 5, 2017.

3. KUOK, Chi-Man et al. Extracorporeal membrane oxygenation support in neonates: a single medical center experience in Taiwan. Pediatrics \& Neonatology, v. 58, n. 4, p. 355-361, 2017.

4. PRINE, Kelli Beckvermit; GORACKE, Kimberly; RUBARTH, Lori Baas. Extracorporeal Membrane Oxygenation in the NICU. Neonatal Network, v. 34, n. 3, p. 183-188, 2015.

5. MOK, Yee Hui et al. Neonatal extracorporeal membrane oxygenation. Advances in Neonatal Care, v. 16, n. 1, p. 26-36, 2016.

6. KATTAN, Javier et al. Neonatal and pediatric extracorporeal membrane oxygenation in developing Latin American countries. J. Pediatr. (Rio J.), v. 93, n. 2, p. 120-129, Apr.2017.Available from <http://www.scielo.br/scielo. php?script=sci_arttext\&pid=S0021- 75572017000200120\&lng=en\&nrm=iso $>$.access

on 12 Sept. 2020. https://doi.org/10.1016/j.jped.2016.10.004.

7. MUGFORD, Miranda; ELBOURNE, Diana; FIELD, David. Extracorporeal membrane oxygenation for severe respiratory failure in newborn infants. Cochrane Database of Systematic Reviews, n. 3, 2008.

8. PAWLIK, Theodore David et al. Medical and financial impact of a neonatal extracorporeal membrane oxygenation referral center in the nitric oxide era. Pediatrics, v. 123, n. 1, p. e17-e24, 2009.

9. LAKATOS, Eva Maria; MARCONI, Marina de Andrade. Técnicas de pesquisa. São Paulo: Atlas, v. 205, 1996.

10. SADDY, Felipe. Estratégias de circulação extracorpórea na Síndrome do Desconforto Respiratório Agudo: uma realidade? Pulmäo RJ, p. 36-43, 2015.

11. VUYLSTEKE, Alain et al. ECMO in the Adult Patient. Cambridge University Press, 2017.

12. DÍAZ, Rodrigo; FAJARDO, Christian; RUFS, Jorge. Historia del ECMO (Oxigenación por membrana extracorpórea o soporte vital extracorpóreo). Revista Médica Clínica Las Condes, v. 28, n. 5, p. 796-802, 2017.

13. GATZWEILER, Eva et al. Extracorporeal membrane oxygenation support in a newborn with lower urinary tract obstruction and pulmonary hypoplasia: a case report. Journal of medical case reports, v. 12, n. 1, p. 210, 2018.

14. LIN, John C. Extracorporeal membrane oxygenation for severe pediatric respiratory failure. Respiratory care, v. 62, n. 6, p. 732-750, 2017.
15. DE OLIVEIRA, Larissa Bertacchini et al. Uso da Membrana de Oxigenação Extracorpórea em uma Paciente Pós-Transplante Pulmonar: Cuidados de Enfermagem. Enfermería Global, v. 14, n. 2, p. 1-32, 2015.

16. VAN OMMEN, Cornelia Heleen; NEUNERT, Cindy E.; CHITLUR, Meera B. Neonatal ECMO. Frontiers in medicine, v. 5, p. 289, 2018.

17. CHAICA, Verónica; PONTÍFICE-SOUSA, Patrícia; MARQUES, Rita. Abordagem de enfermagem à pessoa em situação crítica submetida a oxigenação por membrana extracorporal: Scoping review. Enfermería Global, v. 19, n. 3 , p. 507-546, 2020.

18. SANCHEZ, M. López. Ventilación mecánica en pacientes tratados con membrana de oxigenación extracorpórea (ECMO). Medicina Intensiva, v. 41, n. 8, p. 491-496, 2017.

19. DOS SANTOS, Suelen Maiara et al. Cuidado ao paciente em ECMO (Extracorporeal Membrane Oxygenation): um desafio para a Enfermagem. Semana de Pesquisa da Universidade Tiradentes-SEMPESq, n. 18, 2018.

20. CANÊO, L. F.; NEIROTT, R. A.; ECMO: Improving our Results by Chasing the Rabbits, Brazilian Journal of Cardiovascular Surgery, 30: 657-9, 2015.

22.HARVEY, Chris. Cannulation for neonatal and pediatric extracorporeal membrane

oxygenation for cardiac support. Frontiers in pediatrics, v. 6, p. 17, 2018.

24. BUNGE, Jeroen JH et al. Right ventricular dysfunction during acute respiratory distress syndrome and veno-venous extracorporeal membrane oxygenation. Journal of thoracic disease, v. 10, n. Suppl 5, p. S674, 2018.

25. DALTON, Heidi J. et al. Extracorporeal support in children with pediatric acute respiratory distress syndrome: proceedings from the Pediatric Acute Lung Injury Consensus Conference. Pediatric Critical Care Medicine, v. 16, n. 5_suppl, p. S111-S117, 2015.

26. OUELLETTE, Daniel R. et al. Liberation from mechanical ventilation in critically ill adults: an official American College of Chest Physicians/American Thoracic Society clinical practice guideline: inspiratory pressure augmentation during spontaneous breathing trials, protocols minimizing sedation, and noninvasive ventilation immediately after extubation. Chest, v. 151, n. 1, p. 166-180, 2017.

27. GRAY, B. W.; HAFT, J. W.; HIRSCH, J. C.; ANNICH, G. M.; HIRSCHL, R. B. et al. Extracorporeal life support. Experience with 2,000 patients. ASAIO journal, v. 61, n.1, p. 2-7, 2015. https://doi.org/10.1097/MAT. 0000000000000150.

28. CIANCHI, Giovanni et al. Activities of an ECMO Center for Severe Respiratory Failure: ECMO Retrieval and Beyond, A 4-Year Experience. Journal of cardiothoracic and vascular anesthesia, v. 33, n. 11, p. 3056-3062, 2019. 